\title{
Live-cell nanoscopy enabled with transient labeling and the control of fluorophore blinking
}

\author{
Alexander Mishin*, Maxim Perfilov, Alexey Gavrikov, Anastasia Mamontova, \\ Alexey Bogdanov, and Konstantin Lukyanov \\ Shemyakin-Ovchinnikov Institute of Bioorganic Chemistry of the Russian Academy of Sciences \\ 16/10 Miklukho-Maklaya, Moscow, 117997, Russian Federation
}

\begin{abstract}
Live-cell super-resolution of proteins labeled with genetically encoded fluorescent tags is a challenging task because of the imperfect labeling and the inevitable deterioration of the signal in the course of the experiment. Incomplete maturation of the covalently attached fluorescent tags, inefficient photoconversion, and photobleaching further complicate prolonged live-cell nanoscopy. We have implemented two strategies for lowering the photodamage: ensuring the dynamic replacement of damaged molecules and establishing conditions for the robust intrinsic blinking of the tags at lower illumination powers.
\end{abstract}

Typical super-resolution localization microscopy setup requires illumination powers at the levels harmful to living cells [1]. These harsh conditions are necessary for both squeezing out the maximum possible number of photons from the fluorophore and for the robust blinking of the dye. Alternative means of achieving blinking established for fixed samples, such as the use of strong reductants, are also inadequate for live-cell experiments. Therefore, the photoactivatable and photoswitchable fluorescent proteins (such as Dendra2, mEos3.2, Dronpa, PA-GFP) currently occupy the niche of fluorescent tags for live-cell super-resolution. For that type of probes, the precise control of the number of fluorophores in the light-emitting state is achievable via the additional light source, typically in the nearUV range. We have recently shown that the intrinsic blinking of some genetically encoded proteins can be robust enough even at low-light setup to allow nanoscopy applications [2]. Still, the labeling quality inevitably deteriorates in the course of the experiment due to the depletion of the pool of the available fluorophores. Unfinished maturation of the covalently attached fluorescent marker, incomplete photoconversion, and photobleaching hinder prolonged live-cell nanoscopy and reduce effective labeling density (Fig. 1A).

The general principles of PAINT (Point Accumulation for Imaging in Nanoscale Topography) resolve many challenges in super-resolution microscopy by replenishing the photobleached or defunct dye from the pool of undamaged molecules [3]. We have recently shown the protein-PAINT with a specific transient binding of cell-permeable fluorogenic dyes to genetically encoded protein tags, engineered from bacterial lipocalins [4]. In protein-PAINT, the concentration of externally added chemical dye controls the number of blinking events (Fig. 1B).

\footnotetext{
*Corresponding author: mishin@ibch.ru
} 
While the photon budget of these dyes does not exceed one of the fluorescent proteins, an almost infinite supply of undamaged dyes allows for nanoscopy even at the low-light regime.

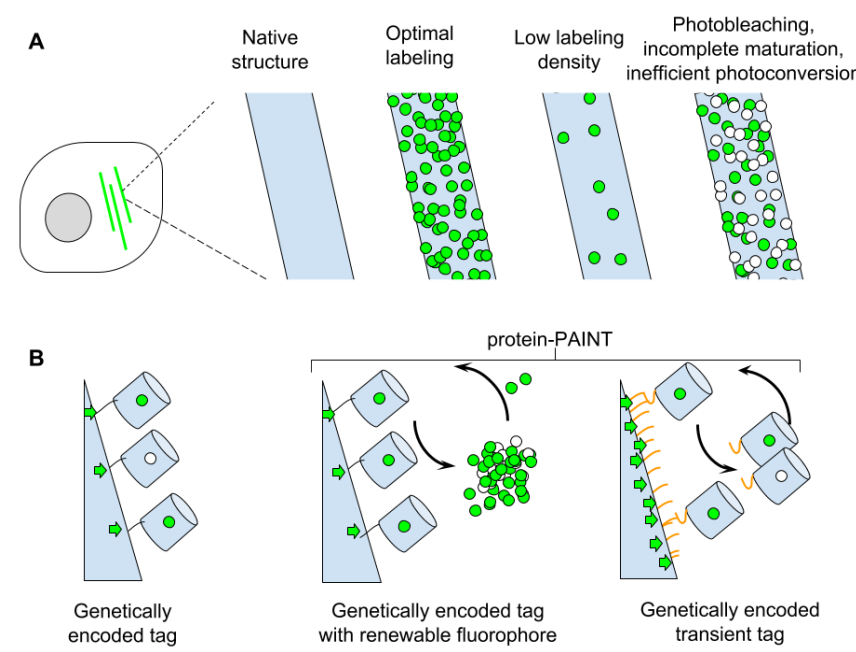

Fig. 1. Challenges of live-cell super-resolution with genetically encoded tags. (A) scheme depicts an enlarged region of the cytoskeleton, the problems in labeling are stated; (B) scheme depicts an enlarged region of a cellular structure, labeled with (left-to-right) a fluorescent protein, the proteinPAINT two-component system with a pool of renewable fluorophores, or with entirely genetically encoded protein-PAINT implementation based on transient protein-protein interactions.

Here we further extend the range of compatible fluorogenic dyes and test other applications of the labeling system, including FRET. We then show the Protein-PAINT implementation based on fluorescent protein and the transient heterodimerization of artificial peptides. Similarly to the protein-PAINT with synthetic dyes, we confirm the improvement in the signal photostability and the labeling density in confocal and superresolution regimes. Also, we provide the data on the effect of the amino acid residues near the chromophore on blinking of photoactivatable green fluorescent proteins. In conclusion, we show how all the techniques mentioned above could be combined to allow prolonged low-light nanoscopy in living cells with minimal photodamage.

This work was supported by the Russian Science Foundation grant 16-14-10364.

\section{References}

1. S. Wäldchen, J. Lehmann, T. Klein, S. van de Linde, M. Sauer, Sci. Rep. 5, 15348 (2015)

2. N. V. Klementieva, A. I. Pavlikov, A. A. Moiseev, N. G. Bozhanova, N. M. Mishina, S. A. Lukyanov, E. V. Zagaynova, K. A. Lukyanov, A. S. Mishin, Chem. Commun., 53, 949 (2017)

3. A. Sharonov, R. M. Hochstrasser. Proc. Natl. Acad. Sci. U.S.A, 103, 18911 (2006)

4. N. G. Bozhanova, M. S. Baranov, N. V. Klementieva, K. S. Sarkisyan, A. S. Gavrikov, I. V. Yampolsky, E. V. Zagaynova, S. A. Lukyanov, K. A. Lukyanov, A. S. Mishin, Chem. Sci, 8, 7138 (2017) 